\title{
Experimental Results of the Fibrin Clot Use to Accelerate the Regeneration of Damaged Bone in the Rat Lower Jaw
}

\author{
I. V. Maiborodin, A. I. Shevela, T. V. Perrin, I. S. Kolesnikov, V. A. Matveeva, \\ A. A. Shevela, B. V. Sheplev, I. A. Kolmakova \\ Center of New Medical Technologies, Institute of Chemical Biology and Fundamental Medicine, \\ Novosibirsk, Russia \\ E-mail: imai@mail.ru \\ Received June 8, 2010; accepted July 13, 2010
}

\begin{abstract}
Morphological and radiological methods were used to study regeneration of the damaged bone of rat mandibles after application of platelet-enriched fibrin clot. A bone hole was artificially created, and in the natural course of regeneration, the hole was immediately filled with blood and there a blood clot formed. After one week of healing, separate islands of young bone tissue appeared. After two to three weeks, the opening in the mandible was completely replaced by the young bone tissue. When a similar bone hole was filled with autological fibrin clot, the blood clot did not form. But after one week the entire hole was filed with newly-formed fused bone tissue. By the second week after the use of fibrin clot, the bone hole had further healed and bone callus was formed.
\end{abstract}

Keywords: Fibrin Clot, Regeneration of Bone, Density of Bone Tissue

\section{Introduction}

Tissue damage leads to the rupture of blood vessels, which, in turn, is the first step of platelet activation after contact with collagen. Platelets initiate the formation of thrombus through the activation of the coagulation system. After the formation of thrombin, fibrinogen is transformed into fibrin, and this is the first step of wound healing. The use of fibrin preparation recreates this process and accelerates healing [1,2].

Initially, fibrin preparation was used in dentistry to accelerate hemostasis after tooth extraction, especially with patients with blood clotting problems. It was also used to close defects in the bone tissue in the maxillofacial region $[3,4]$.

Then, fibrin glue was used in lieu of sutures to attach tissue during operations, and to improve the grafting of implants from artificial and synthetic materials $[5,6]$.

Platlet-rich plasma is a modification of the fibrin glue prepared from autologous blood and containing a set of cytokines, which causes the migration and division of all mesenchymal (including chondrocytes and mesenchymal stem cells) and epithelial cells, and stimulates the synthesis of collagen and the matrix of connective tissue $[7,8]$.
When fibrin degrades, it causes a migration of osteogenic cells and gingival fibroblasts in vitro and more rapid regeneration of surgical bone defects in the experiment in vivo. Fibrin glues and films can serve as a substrate to support the growth of fibroblasts and their functions. Thus, the adhesive material containing fibrin and fibronectin, their monomers or degradation products, accelerate the healing of periodontal tissue, including bone tissue $[9,10]$.

Compared to the natural course of healing, applying of platelet-enriched fibrin clot (PEFC) results in less pronounced acute and chronic inflammation in damaged tissues. The alteration phase very rapidly replaced by regenerative-reparative processes. Therefore, application of fibrin materials can be used to accelerate tissue regeneration and to facilitate the grafting of implants in experiments and in the clinic [11-13].

It should be noted that along with the positive results of the use of preparations of fibrin, there is evidence of the ineffectiveness of these therapies in dentistry [14-16].

Thus, the literature contains many contradictory and mutually exclusive data on the effectiveness of the use of fibrin preparations in surgery and dentistry. However, these studies do not reflect the use of fibrin for regenerating bone tissue, and in particular, PEFC prepared from autologous blood plasma with platelets. 


\section{Aim}

By using morphological and radiological methods, the natural course of healing and the result of PEFC application to the regeneration of the damaged rat lower jaw were compare in experiment.

\section{Material and Methods}

The experiment were used 6 month-old Wag male-rats weighing 180-200g. All procedures on the rats were performed under general anesthesia of ether inhalation in a sanitary operating environment in compliance with the "Regulations of the work using experimental animals." At every point of the study, at least 6 rats were used.

It was decided to create holes in bone tissue, which has few individual differences (especially blood vessels and nerves), and does not move when muscles are moving. The lower jaw was chosen due to the fact that there is enough strength and width of the bone combined with ease of access. In addition, the rats cannot prematurely tear out their stitches.

Preparation of PEFC: Several rats of the same breed were decapitated, and 2-7 $\mathrm{ml}$ of blood was collected in sterile glass tubes. This blood was centrifuged at 2800 rpm for 12 minutes $[11,13]$. Then the upper part (platelet-rich fibrin clot or fibrin clot with platelet) was placed in sterile Petri dishes and maintained for several hours in an incubator at $37^{\circ} \mathrm{C}$ until use. Then, immediately before use, sterile scissors were used to cut the PEFC fragments to the correct sizes.

Creation of the Bone Defect and PEFC Application in the Experiment: Under general inhalant ether anesthesia, in a clean operating room, while respecting the rules of asepsis and antisepsis, after treating the skin with $70 \%$ alcohol, a skin incision was made using a scalpel. The incision was $1.5-2 \mathrm{~cm}$ along the bottom edge of the mandible. Retractors were used to detach the masticatory muscles and expose the lower surface of the lower jawbone. A dental drill was used at a specific manner (same size, even edges, depth control, the same rotational speed and, consequently, heating of tissues and the possibility of cooling) to create a $2 \mathrm{~mm}$ in diameter round hole through the bone in region of mandibular angle, the hole did not connect with the oral cavity. In the control group of rats (natural healing), the bone defect was covered with masticatory muscle and then simple running sutures were used on the skin, and it was treated with alcohol. In the other group, forceps were used to fill the holes with PEFC. The size of the PEFC was slightly larger than the holes. After packing the bones with PEFC, the bone defects were also covered with the masticatory muscle, the skin was sutured with continuous sutures, and alcohol was applied to the wound. All implant materials were sterile.
Animals were withdrawn from the experiment after 1 , 2, 3, 4 and 5 weeks after surgery. The bone tissue with defects in the mandible was studied.

Fragments of the mandible were preserved in a $4 \%$ paraformaldehyde on biphosphate buffer ( $\mathrm{pH}$ 7.4) for at least 24 hours. After preservation, the skin, subcutaneous tissue and chewing muscles were removed. The fragments of mandibles were decalcified in solution "Biodek R" (Bio Optica Milano, Italy) for 24 hours, dehydrated in a gradien of ethanol, lightened in xylene, and embedded in paraffin. Sections of 5-7 microns thick were stained with hematoxylin and eosin, and studied under a light microscope Axioimager M1 (Carl Zeiss, Germany) with a magnification of up to 1200 times.

Radiological studies were performed to observe the reparative processes in the mandibles of experimental animals at various healing intervals (Figures 1 and 2). The tissue density was estimated in the hole itself, and in the contralateral part of the mandible.

Statistical analysis was performed using applied statistical program of MS Excel 7.0 (Microsoft, USA). The arithmetic mean and standard deviation were determined, the differentiation between means was considered significant at $\mathrm{p} \leq 0.05$, used the Student's criterion.

\section{Results}

At 1 week after injury to the bones of the rat lower jaw with natural recovery, it was found that the hole was partially filled with blood and the hole contained some fragments of connective tissue and granulation (Figure 3). This marked the beginning of bone formation in the defect (formation of separate islands of young bones and cartilage among granulation) (Figure 3).

After 2 weeks the hole was completely closed by the young bone tissue with a large number of blood vessels on the edge of the defect. Cartilage tissue was also present among the newly formed bone structures.

At 3 weeks the hole was completely closed by the newly formed bone tissue. The only evidence that the defect existed were the remaining large vessels and randomly located bone trabeculae (bone callus). At this point, a fully formed cavity with bone marrow appeared.

After 4 and 5 weeks, in most cases, the only remaining trace of the operation was bone callus (Figure 4).

One week after injury to the bone with use of PEFC, the hole was completely filled with fused islets of newly formed bone (Figure 5). In other words, after the application of PEFC, bone regeneration resulted in the complete filling of the artificial hole after one week.

In most cases, after two weeks, the injury to the bone was filled, regardless of whether PEFC was applied. The holes were closed with newly-formed bone tissue with a large number of blood vessels at the periphery of the defect and cartilage tissue in the center. 


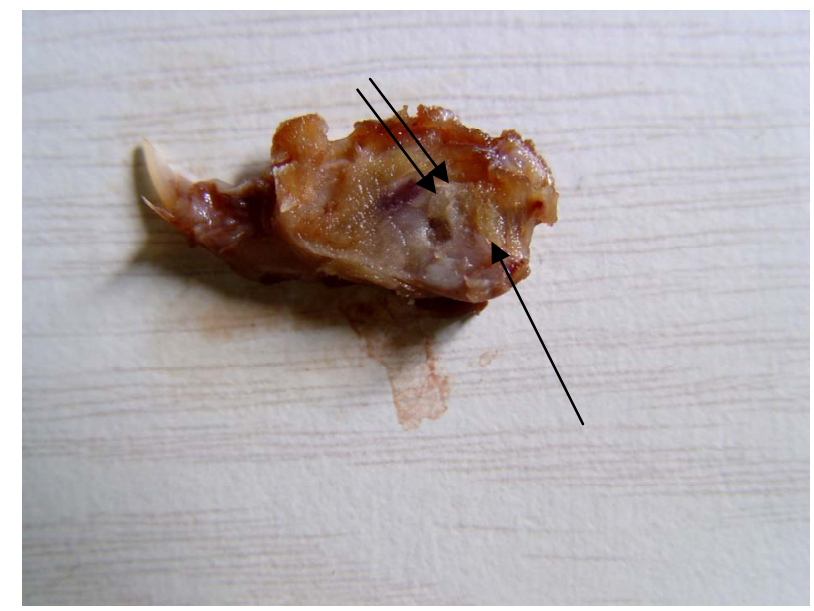

Figure 1. Macroscopic appearance of rat mandible with remote masticatory muscles 1 week after injury and natural regeneration. There is no evidence of purulent inflammatory process. An arrow indicates the artificially created opening filled with blood clot. The two arrows- the root of the central incisor.

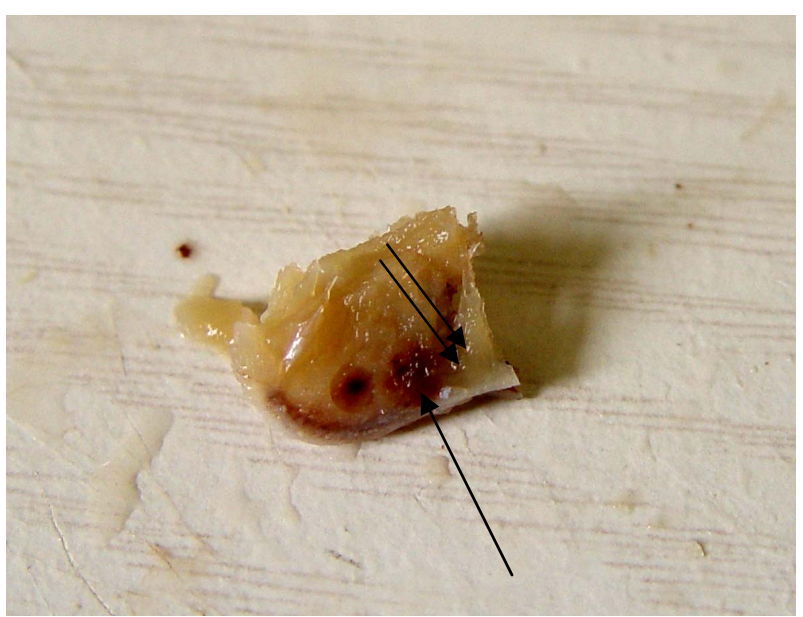

Figure 2. Macroscopic fragment of mandible of rat 1 week after a bone defect is then filled with PEFC. The artificially created hole has no macroscopic signs of inflammation, is filled, and is located at the level of the surrounding tissues. An arrow indicates the artificially created hole filled with PEFC. The two arrows- the root of the central incisor.

After 3, 4 and 5 weeks of healing, the hole was completely covered by newly formed bone tissue with randomly arranged bone trabeculae formed callus and cavities with bone marrow (Figure 6). This was true in the mandibles that healed naturally and the ones with PEFC applied.

After statistically controlling for data of densitometry of the rats' mandibles' bone defect regeneration in natural healing and after applying PEFC, no significant differences in the density of tissue between the compared groups of animals was found at each point of the study. However, the density of tissue in the natural reparative processes was statistically significantly different from healthy bone on the contralateral side during the first 3 weeks. In contrast, the density of tissue in the PEFC assisted process was statistically significantly different from healthy bone on the contralateral side only during the first and second weeks. (Figures 7 and 8) (Table 1). That is, the bone tissue with PEFC applied became dense faster than with natural healing.

In addition, it should be noted that all periods of observation the density of tissue in the affected area after applying PEFC was slightly higher than during the natural course of repair (Figures $\mathbf{7}$ and 8 ), although this difference was not significant. The maximum difference in tissue density was noted from 2 to 4 weeks. By week 5 , the differences were smoothed out somewhat (Table 1).

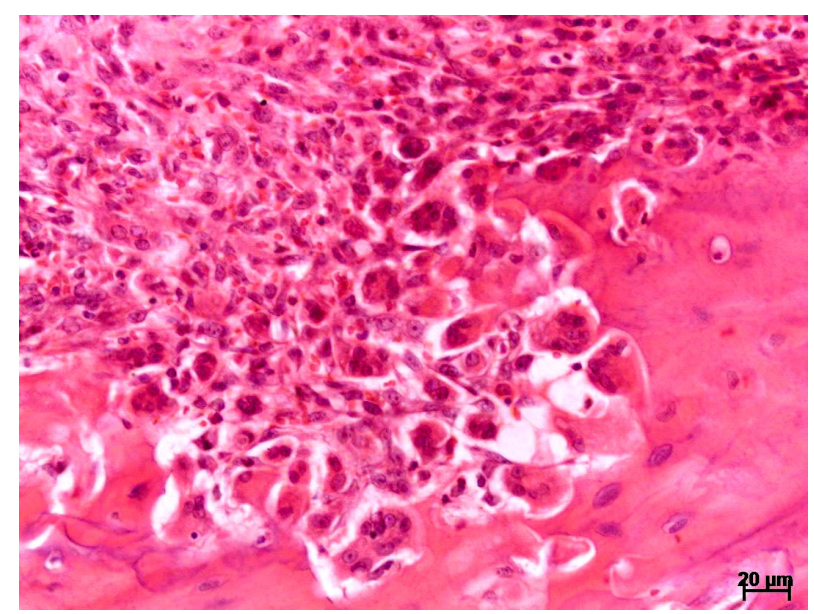

Figure 3. The formation of bone structures at the periphery of the damaged part of the mandible in natural healing 1 week after surgery. Hematoxylin and eosin.

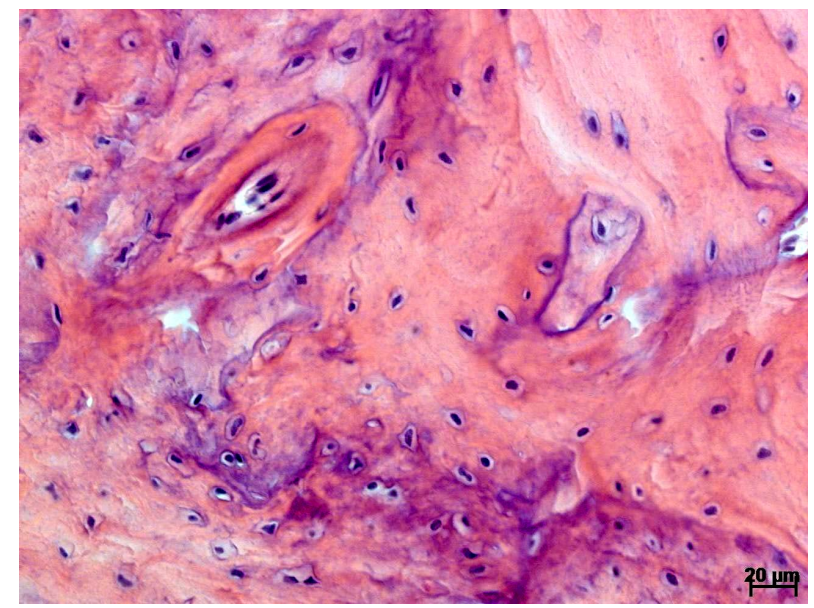

Figure 4. Bone defect of the mandible with self-regeneration 4 weeks after surgery. Bone tissue struts in the callus are unstructured. Hematoxylin and eosin. 


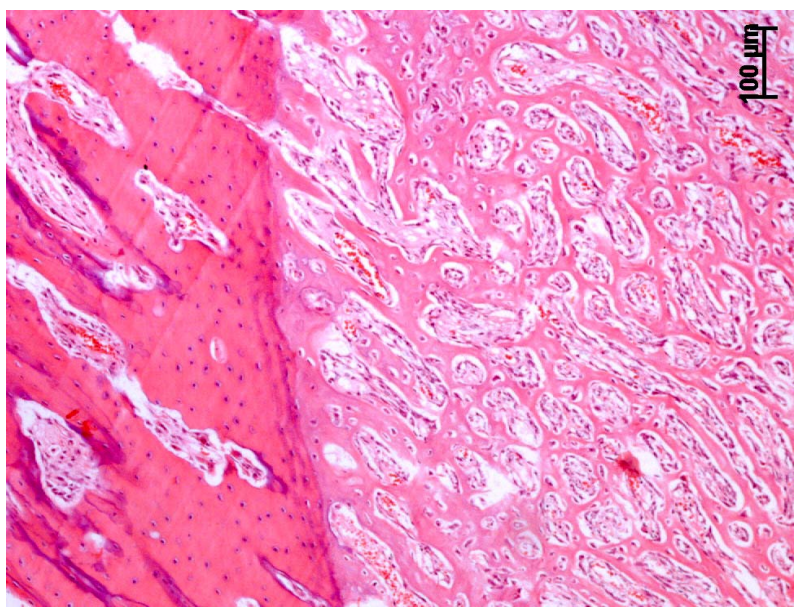

Figure 5. Healing area of damaged bone of the mandible 1 week after surgery using PEFC. Bone defect filled with fused islands of the young bone tissue with a large number of vessels. Hematoxylin and eosin.

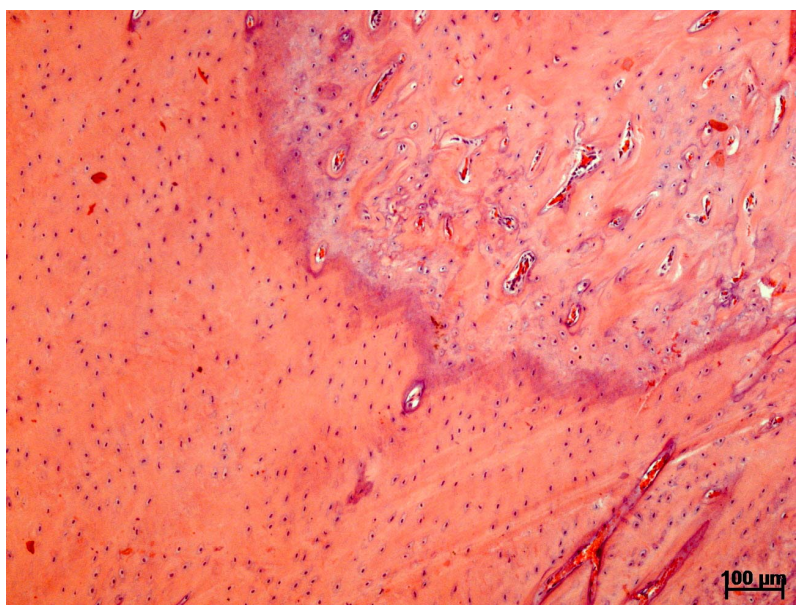

Figure 6. Structures of callus on the spot of the holes in the bones of the mandible 4 weeks after the operation and use PEFC. Hematoxylin and eosin.

\section{Discussions}

In the experiment, when the bone tissue was damaged, an acute inflammation of the tissues occurred. This process occurs in response to direct tissue damage as a result of surgical intervention. Over time, an inflammatory reaction due to the operation subsides, and the process of restoration of damaged tissues begins.

During the natural healing process, when the mandibles were damaged, the holes immediately filled with blood, and a clot formed with a large number of red blood cells. Gradually, this clot was dissolved by phagocytes (first neutrophils, then macrophages), and was gradually replaced by migrating osteogenic cells. Due to the functioning of osteoblasts, the young bone tissue began to take shape from the edges of the defect. These islands of young bone become wider and merge. In almost all cases, 2-3 weeks in rats, a complete bone regeneration took place in the artificially created defects. It should be noted that the morphological data on bone regeneration by the dates specified were confirmed by the results of densitometry.

Fibrin in tissue, according to published data, reduces the severity of the inflammatory process [11-13] and limits the spread of infection $[17,18]$. That is, the introduction of PEFC in the cavity of the wound, apparently, can protect the surrounding tissues from the dissemination of microorganisms, and from excessive exposure of lysosomal enzymes of phagocytes. This limits tissue destruction and, therefore, earlier starting the regenerative processes in tissues, there is less antigen and detritus, and a more rapid cleansing of the wound.

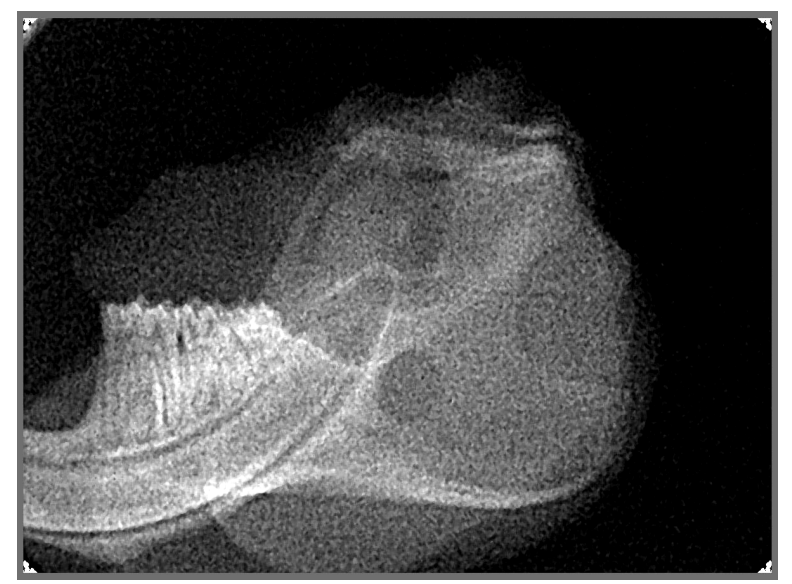

Figure 7. Bone defect of the mandible during the natural course of recovery 3 weeks after the operation, according to radiological study, artificially created hole (indicated by arrow) is preserved.

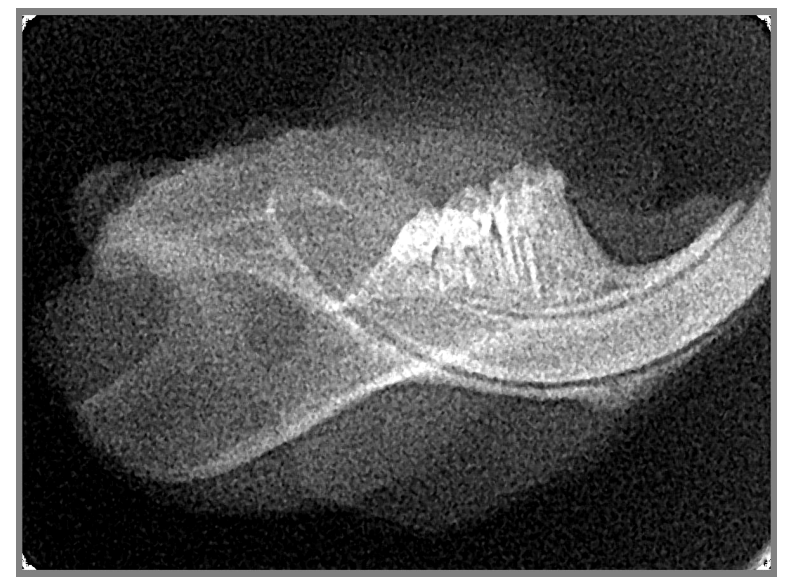

Figure 8. Artificially created opening in the bones of the lower jaw (indicated by arrows) is retained according radiological study 3 weeks after surgery using PEFC. The density of tissue in the defect after the application PEFC above. 
Table 1. The bone density in defect of the lower jaw in comparison with surrounding intact tissues $(S \pm \sigma)$.

\begin{tabular}{|c|c|c|c|}
\hline \multirow{2}{*}{$\begin{array}{l}\text { Time after } \\
\text { operation }\end{array}$} & \multicolumn{2}{|c|}{ Regeneration Process } & \multirow{2}{*}{$\begin{array}{c}\text { Difference in } \\
\text { Density (Fibrin- } \\
\text { Control) in Defect }\end{array}$} \\
\hline & Natural Healing & $\begin{array}{l}\text { After Using } \\
\text { PEFC }\end{array}$ & \\
\hline 1 Week & $0.892 \pm 0$. & $.913 \pm 0.017^{*}$ & $0.021 \pm 0.05$ \\
\hline 2 Weeks & $0.922 \pm 0.038^{*}$ & $0.953 \pm 0.021^{*}$ & $0.031 \pm 0.033$ \\
\hline 3 Weeks & $0.914 \pm 0.033 *$ & $0.949 \pm 0.036$ & $0.035 \pm 0.051$ \\
\hline 4 Weeks & $0.912 \pm 0.059$ & $0.942 \pm 0.048$ & $0.03 \pm 0.043$ \\
\hline 5 Weeks & $0.913 \pm 0.064$ & $0.924 \pm 0.063$ & $0.011 \pm 0.008$ \\
\hline
\end{tabular}

Note: * - data, significantly different from the intact bone on the contralateral side $(\mathrm{p} \leq 0.05)$.

In addition, the fibrin clot acts as matrix capturing migrating leukocytes (neutrophils), endotheliocytes and fibroblasts [7-10]. Thrombospondin-1 from platelets stimulates tubulogenez (initial stage of angiogenesis) by endothelial cells [10].

Migrating through fibrin $[7,8]$, neutrophils more rapidly reach all sections of the wounds, even wounds covered with layers of pus and detritus and, thus, tissues are more rapidly cleared from the antigenic substances (microorganisms and the same detritus). In addition, when moving through fibrin clot neutrophils partially dilute it with its own enzymes, so even dense fibrin clots become less dense, and similar to a net.

Fibroblasts, located in the fibrin network $[7,8,10]$, begin the synthesis of collagen, not only from the bottom of the wound, but also from its cavity, thus the scar tissue forms more rapidly.

It should be noted that the fibrin not only facilitates the migration of fibroblasts, but it also accelerates the synthesis of connective tissue [5,7,8,11-13,20].

Fibrin also stimulates the migration of endotheliocytes [7-9], and therefore the process of angiogenesis begins more quickly [19]. The newly formed blood vessels are located not only in the granulations on the wound bottom, but also in the fibrin net. The more rapid growth of blood vessels, in turn, facilitates migration of leukocytes from the blood vessels and synthesis of components of connective tissue.

When the bone injuries were filled with PEFC, there was no need to wait for the blood clot will be destroyed and the red blood cells will be eliminated via through phagocytosis. After one week in most cases, the bone defect was already filled with fused islets of newlyformed bone tissue. That is, when PEFC was applied, the artifical defect was almost completely filled after one week.

By the second week after using PEFC there was a further gradual filling of newly formed bone tissue in the defect with a large number of blood vessels in the periphery. By third week the formation of bone callus completely covered the opening of the bone, also red bone marrow was observed in the defect. These changes continued to occur to varying degrees in subsequent periods of observation.

Fibrin is present in both natural healing and in the PEFC enhanced process. Fibrin facilitates the migration of neutrophils, endothelial cells, macrophages, osteoblasts and other cellular elements. However, what distinguishes natural healing is the large number of red blood cells in the blood clot. The presences of these cells in the fibrin net impede the migration of the aforementioned cellular elements. In addition, some potential phagocytes will be spent not only on the intake of detritus, but also on phagocytosis of red blood cells from a clot.

Thus, on the basis of the foregoing, we conclude that when PEFC is applied, the start of the repair processes is more intense than in the spontaneous healing. The hole in the bone quickly filled with islands of bone tissue, which merged earlier than in the natural process. Apparently, the formation of the young bones begins immediately after the operation without the need to spend time for the process of lysis and the removal of red blood cells from the clot.

Since application of PEFC causes a more intense regeneration of damaged bone, it appears to be advisable to use PEFC to accelerate the reparative processes of bone tissue in dentistry, surgery and traumatology.

This work was financial supported by the fundamental research program of the Presidium of RAS "Fundamental Science - Medicine" (project № 21.31 "Development of technologies for process management of bone tissue regeneration using biodegradable polymers").

\section{Conclusions}

In the natural course of regeneration, when the mandibles of rats were damaged, the defect was filled with a blood clot with a large number of red blood cells. After 1 week of healing, the damaged area contained separate islands of young bone tissue, as well as fragments of the blood clot and granulation. After 2-3 weeks of healing, the opening in the bone of the lower jaw was completely replaced by the young bone tissue.

When the side of damaged rat mandibles was filled with PEFC, no blood clot was formed. After one week, the entire bone defect was filled with newly formed islets fused bone. By the second week after using PEFC there was a further substitution of the defect with bone tissue and the formation of bone callus.

\section{References}

[1] M. E. Laidmae, J. L. McCormick, J. J. Herod, T. Pastore, E. S. Salum, P. A. Sawyer, Janmey and R. Uibo, "Stability, Sterility, Coagulation, and Immunologic Studies of Salmon Coagulation Proteins with Potential Use for Mammalian Wound Healing and Cell Engineering," 
Biomaterials, Vol. 27, No. 34, 2006, pp. 5771-5779.

[2] M. Valbonesi, "Fibrin Glues of Human Origin," Best Practice \& Research Clinical Haematology, Vol. 19, No. 1, 2006, pp. 191-203.

[3] G. Carter, A. Goss, J. Lloyd and R. Tocchetti, "Tranexamic Acid Mouthwash Versus Autologous Fibrin Glue in Patients Taking Warfarin Undergoing Dental Extractions: A Randomized Prospective Clinical Study," Journal of Oral and Maxillofacial Surgery, Vol. 61, No. 12, 2003, pp. 1432-1435.

[4] W. D. Spotnitz and R. Prabhu, "Fibrin Sealant Tissue Adhesive-Review and Update," Journal of Long-Term Effects of Medical Implants, Vol. 15, No. 3, 2005, pp. 245-270.

[5] W. Becker, "Fibrin Sealants in Implant and Periodontal Treatment: Case Presentations," Compend Contin Educ Dent, Vol. 26, No. 8, 2005, pp. 539-545.

[6] J. Choukroun, A. Diss, A. Simonpieri, M. O. Girard, C. Schoeffler, S. L. Dohan, A. J. Dohan, J. Mouhyi and D. M. Dohan, "Platelet-Rich Fibrin (PRF): A Second-Generation Platelet Concentrate. Part IV: Clinical Effects on Tissue Healing," Oral Surgery, Oral Medicine, Oral Pathology, Oral Radiology, Vol. 101, No. 3, 2006, pp. e56e60.

[7] M. B. Schmidt, E. H. Chen and S. E. Lynch, "A Review of the Effects of Insulin-Like Growth Factor and Platelet Derived Growth Factor on in Vivo Cartilage Healing and Repair," Osteoarthritis Cartilage, Vol. 14, No. 5, 2006, pp. 403-412.

[8] D. Schwartz-Arad, L. Levin and M. Aba, "The Use of Platelet Rich Plasma (PRP) and Platelet Rich Fibrin (PRF) Extracts in Dental Implantology and Oral Surgery," Refuat Hapeh Vehashinayim, Vol. 24, No. 1, 2007, pp. 51-55, 84 .

[9] E. L. Kaijzel, P. Koolwijk, M. G. vanErck, V. W. vanHinsbergh and M. P. deMaat, "Molecular Weight Fibrinogen Variants Determine Angiogenesis Rate in a Fibrin Matrix in Vitro And in Vivo," Journal of Thrombosis and Haemostasis, Vol. 4, No. 9, 2006, pp. 1975-1981.

[10] S. McDougall, J. Dallon, J. Sherratt and P. Maini, "Fibroblast Migration and Collagen Deposition During Dermal Wound Healing: Mathematical Modelling and Clinical Implications," Philosophical Transactions Mathematical Physical and Engineering Sciences, Vol. 364, No. 1843, 2006, pp. 1385-1405.

[11] I. V. Maiborodin, I. S. Kolesnikov, B. V. Sheplev, T. M. Ragimova, A. I. Shevela, A. N. Kovyntsev, I. A. Komakova, I. A. Pritchina, E. V. Kozlova, A. B. Voitovich and D. N. Kovyntsev, "Granulomatous Inflammation after Use Fibrin Preparation," Morphological Letters, No. 3-4, 2007, pp. 116-118.

[12] I. V. Maiborodin, I. S. Kolesnikov, B. V. Sheplev and T. M. Ragimova, "Application of Fibrin and its Preparations in Stomatology," Stomatologiia (Mosk), Vol. 87, No. 6, 2008, pp. 75-77.

[13] I. V. Maiborodin, I. S. Kolesnikov, B. V. Sheplev, T. M. Ragimova, A. N. Kovyntsev, D. N. Kovyntsev and A. I. Shevela, "Adjusting Gingival Tissues Morphology After Dental Implantation with Fibrin Use," Stomatologiia (Mosk), Vol. 88, No. 1, 2009, pp. 9-13.

[14] S. J. Froum, S. S. Wallace, D. P. Tarnow and S. C. Cho, "Effect of Platelet-Rich Plasma on Bone Growth and Osseointegration in Human Maxillary Sinus Grafts: Three Bilateral Case Reports," The International Journal of Periodontics \& Restorative Dentistry, Vol. 22, No. 1, 2002, pp. 45-53.

[15] G. Fuerst, R. Gruber, S. Tangl, F. Sanroman and G. Watzek, "Effects of Fibrin Sealant Protein Concentrate with and Without Platelet-Released Growth Factors on Bony Healing of Cortical Mandibular Defects, an Experimental Study in Minipigs," Clinical Oral Implants Research, Vol. 15, No. 3, 2004, pp. 301-307.

[16] R. M. London, F. A. Roberts, D. A. Baker, M. D. Rohrer and R. B. O'Neal, "Histologic Comparison of a Thermal Dual-Etched Implant Surface to Machined, TPS, and HA Surfaces: Bone Contact in Vivo in Rabbits," The International Journal of Oral \& Maxillofacial Implants, Vol. 17, No. 3, 2002, pp. 369-376.

[17] J. Choukroun, A. Diss, A. Simonpieri, M. O. Girard, C. Schoeffler, S. L. Dohan, A. J. Dohan, J. Mouhyi and D. M. Dohan, "Platelet-Rich Fibrin (PRF): A Second-Generation Platelet Concentrate. Part V: Histologic Evaluations of PRF Effects on Bone Allograft Maturation in Sinus Lift," Oral Surgery, Oral Medicine, Oral Pathology, Oral Radiology \& Endodontics, Vol. 101, No. 3, 2006, pp. 299-303.

[18] D. M. Dohan, J. Choukroun, A. Diss, S. L. Dohan, A. J. Dohan, J. Mouhyi and B. Gogly, "Platelet-Rich Fibrin (PRF): A Second-Generation Platelet Concentrate. Part III: Leucocyte Activation: A New Feature for Platelet Concentrates?" Oral Surgery, Oral Medicine, Oral Pathology, Oral Radiology \& Endodontics, Vol. 101, No. 3, 2006, pp. e51-e55.

[19] S. Kellouche, S. Mourah, A. Bonnefoy, D. Schoevaert, M. P. Podgorniak, F. Calvo, M. F. Hoylaerts, C. Legrand and C. Dosquet, "Platelets, Thrombospondin-1 and Human Dermal Fibroblasts Cooperate for Stimulation of Endothelial Cell Tubulogenesis through VEGF and PAI-1 Regulation," Experimental Cell Research, Vol. 313, No. 3, 2007, pp. 486-499.

[20] K. Ito, Y. Yamada, T. Naiki and M. Ueda, "Simultaneous Implant Placement and Bone Regeneration Around Dental Implants Using Tissue-Engineered Bone with Fibrin Glue, Mesenchymal Stem Cells and Platelet-Rich Plasma," Clinical Oral Implants Research, Vol. 17, No. 5, 2006, pp. 579-586. 